This is the author's final, peer-reviewed manuscript as accepted for publication. The publisher-formatted version may be available through the publisher's web site or your institution's library.

\title{
DDE and PCB serum concentration in maternal blood and their adult female offspring
}

Wei-Wen Hsu, Janet Rose Osuch, David Todem, Bonita Taffe, Michael O'Keefe, Selamawit Adera, Wilfried Karmaus

\section{How to cite this manuscript}

If you make reference to this version of the manuscript, use the following information:

Hsu, W.-W., Osuch, J. R., Todem, D., Taffe, B., O'Keefe, M., Adera, S., \& Karmaus, W. (2014). DDE and PCB serum concentration in maternal blood and their adult female offspring. Retrieved from http://krex.ksu.edu

\section{Published Version Information}

Citation: Hsu, W.-W., Osuch, J. R., Todem, D., Taffe, B., O'Keefe, M., Adera, S., \& Karmaus, W. (2014). DDE and PCB serum concentration in maternal blood and their adult female offspring. Environmental Research, 132, 384-390.

Copyright: @ 2014 Elsevier Inc

Digital Object Identifier (DOI): doi:10.1016/j.envres.2014.03.009

Publisher's Link: http://www.sciencedirect.com/science/article/pii/S0013935114000553

This item was retrieved from the K-State Research Exchange (K-REx), the institutional repository of Kansas State University. K-REx is available at http://krex.ksu.edu 


\section{DDE and PCB serum concentration in maternal blood and their adult female offspring}

Wei-Wen Hsu a, Janet Rose Osuch ${ }^{\text {b, }}$, David Todem ${ }^{\text {c }}$, Bonita Taffe ${ }^{\mathrm{d}}$, Michael O’Keefe ${ }^{\mathrm{d}}$, Selamawit Adera ${ }^{\mathrm{e}}$, Wilfried Karmaus ${ }^{\mathrm{e}}$

${ }^{a}$ Department of Statistics, Kansas State University, Manhattan, Kansas, USA

${ }^{\mathrm{b}}$ Departments of Surgery and Epidemiology and Biostatistics, Michigan State University, East Lansing, Michigan, USA

${ }^{c}$ Department of Epidemiology and Biostatistics, Michigan State University, East Lansing, Michigan, USA

${ }^{\mathrm{d}}$ Bureau of Laboratories, Michigan Department of Community Health, Lansing, Michigan, USA

${ }^{\mathrm{e}}$ Division of Epidemiology, Biostatistics, and Environmental Health, School of Public Health, University of Memphis, Memphis, Tennessee, USA

* Corresponding Author:

Janet Osuch, Professor

Departments of Surgery and Epidemiology and Biostatistics

West Fee Hall, 909 Fee Road, Room 632

College of Human Medicine

Michigan State University

East Lansing, Michigan 48824

Phone: 517-353-5440 extension 1

Fax: 517-432-1130

Email: janet.osuch@hc.msu.edu 
Keywords: intra-uterine exposure, dichlorodiphenyl dichloroethylene, polychlorinated biphenyls, sport-caught fish consumption, female offspring

\title{
Human subjects research
}

The investigations were approved prior to their inception by the Institutional Review Boards at Michigan State University and the Michigan Department of Community Health (MDCH) and renewed at yearly intervals.

\author{
Abbreviations \\ BMI: $\quad$ Body mass index \\ DDE: $\quad$ Dichlorodiphenyl-dichloroethylene \\ ICC: $\quad$ Intraclass correlation coefficient \\ LOD: $\quad$ Limit of detection \\ MDCH: $\quad$ Michigan Department of Community Health \\ OC Organochlorines \\ PCBs: $\quad$ Polychlorinated biphenyls
}




\begin{abstract}
Background: Dichlorodiphenyl dichloroethylene (DDE) and polychlorinated biphenyls (PCBs) can be passed from mother to offspring through placental transfer or breast feeding. Unknown is whether maternal levels can predict concentrations in adult offspring.
\end{abstract}

Objectives: To test the association between maternal blood levels of DDE and PCBs and adult female offspring levels of these compounds using data from the Michigan Fisheaters'Cohort.

Methods: DDE and PCB concentrations were determined in 132 adult daughters from 84 mothers. Prenatal exposures were estimated based on maternal DDE and PCB serum levels measured between 1973 and 1991. Levels in adult daughters were regressed on maternal and estimated prenatal exposure levels, adjusting for potential confounders using linear mixed models. Confounders included daughter's age, birth order, birth weight, number of pregnancies, the length of time the daughter was breast-fed, the length of time the daughter breast-fed her own children, last year fish-eating status, body mass index, and lipid weight.

Results: The median age of the participants was 40.4 years (range 18.4 to $65.4,5-95$ percentiles 22.5-54.6\%, respectively). Controlling for confounders and intra-familial associations, DDE and PCB concentrations in adult daughters were significantly positively associated with estimated prenatal levels and with maternal concentrations. The proportion of variance in the adult daughters' organochlorine concentrations explained by the maternal exposure levels is approximately $23 \%$ for DDE and $43 \%$ for PCBs. The equivalent of a median of $3.67 \mu \mathrm{g} / \mathrm{L}$ prenatal DDE and a median of $2.56 \mu \mathrm{g} / \mathrm{L}$ PCBs were 15.64 and 10.49 years of fish consumption, respectively. When controlling for effects of the shared environment (e.g., fish diet) by using a subsample of paternal levels measured during the same time frames $(n=53$ and $n=37)$, we determined that the direct maternal transfer remains important.

Conclusions: Estimated intrauterine DDE and PCB levels predicted concentrations in adult female offspring 40 years later. Interpretation of adverse health effects from intrauterine exposures of persistent pollutants may need to consider the sustained impact of maternal DDE and PCB levels found in their offspring. 


\section{Introduction}

Endocrine-disrupting chemicals, including the organochlorines (OC) dichlorodiphenyltrichloroethylene (DDT), its metabolite dichlorodiphenyl-dichloroethylene (DDE) and polychlorinated biphenyls (PCBs), were used for a variety of purposes after their introduction following World War II (Mnif et al., 2011). Both were banned in the 1970s in the United States because of concern about adverse health consequences. The epidemiologic literature in humans has subsequently documented links between OCs and a variety of human health conditions, including cancer, obesity, diabetes, the metabolic syndrome, and a variety of reproductive outcomes, including effects on the menstrual cycle, duration of breastfeeding, and decreased fertility in men (De Coster and van Larebeke, 2012).

Many women exposed to these chemicals when circulating blood levels were at their highest were of reproductive age, and because many of these chemicals have been shown to cross the placenta, their fetuses became exposed as well. Several studies examining the correlation of maternal and fetal serum concentrations of these chemicals demonstrate stronger associations between levels of DDE than PCBs, and some PCB congeners do not appear to cross the placenta (Adetona et al., 2013; Butler Walker et al., 2003; DeKoning and Karmaus, 2000; Kanja et al., 1992; Porpora et al., 2013; Sala et al., 2001; Waliszewski et al., 2001).

No human study has yet investigated whether maternal serum levels of DDE and PCBs can be used to predict levels of these substances in adult offspring. The answer to this question has public health and research implications. From a public health perspective, mothers with a higher exposure in the past may significantly add to baseline levels in their adult offspring, contributing to potential adverse health effects such as asthma (Gascon et al., 2013), diabetes (Wu et al., 2013), and endocrine disorders (Langer), to name a few. 
From a research and scientific standpoint, the relevance pertains to compelling theories regarding the developmental origin of adult diseases (Bezek et al., 2008). Xenobiotics have the potential to influence cellular development and/or function during critical periods of fetal development and thus may permanently alter the structure or function of specific organ systems, leading to long-term health problems. However, some theories regarding intrauterine exposures neglect the possibility that the toxic substance itself may be passed from mother to offspring, leading to an additional opportunity for predisposition to disease from the exposure. It is possible that transfer of deleterious chemicals from mother to offspring, either through the placenta or via breast milk, may explain at least some of the blood OC adult levels of their offspring. If so, these levels may in turn continue to produce adverse effects that are mistakenly attributed solely to prenatal exposures.

The overall hypothesis of this study is that after, 40 years on average, gestational concentrations of DDE and PCBs will be significantly positively associated with the average adult DDE and PCB levels in female offspring.

\section{Materials and Methods}

\subsection{Study Population and Recruitment}

Mothers and participating adult daughters were identified from the Michigan Fisheaters' Cohort, established by the Michigan Department of Community Health (MDCH) between 1973 and 1991 for purposes of studying the health effects of OC exposure in anglers and their families who consumed sport-caught fish (Humphrey, 1983).

To establish this cohort, participant recruitment originally took place in eleven counties along the shoreline of Lake Michigan at sites of fishing activities (e.g., docks, marinas, and bait 
shops) at three different time points between 1973 and 1991. During the first, (1973-1974), 156 participants were recruited and their serum PCB levels were determined. The second period (1979-1982) yielded 1,140 new participants and 115 of 156 (73.7\%) from the previous period; both DDE and PCB serum levels were determined. The third period (1989-1991) reenrolled 717 participants from the combined 1255 enrolled in the second period (57.1\%), and recruited 11 new participants, for a total recruitment of 728 participants constituting the parental (F0) generation. Questionnaires for the parental population were administered at each time point and included information about the number and gender of offspring as well as a request for permission to re-contact the participants for further studies. All information was stored in a protected electronic database.

In 2000, the Michigan Department of Community Health (MDCH) conducted a follow- up mailing to these participants. Vital statistics data was used to exclude 42 known deceased participants. Letters were mailed to 686 individuals constituting 621 families. Forty-six individuals were unable to provide follow-up data because of medical conditions (unable to communicate secondary to stroke or dementia, etc), 90 could not be located, 125 declined participation, and 27 additional were deceased. Three hundred ninety-eight participants provided answers to the mailing. In 2001/2002, after institutional approval, we re-contacted this group for the sole purpose of asking them to provide information about the number and location of their offspring. We identified individuals from this parental group who met the following eligibility criteria: 1) were female, 2) had given birth to a daughter between 1950 and 1980 (so that the daughters were 15-45 years of age), and 3) had PCB and DDE levels measured at least once. One hundred and one women in the F0 generation met these criteria; they gave birth to 213 living daughters aged $15-45$ years during the eligibility period. 
In 2006/07, the 213 daughters received a mailing containing a newsletter about results of the Michigan Fisheaters' Cohort, and a brochure explaining a new study. The investigations were approved by the Institutional Review Boards at Michigan State University and the MDCH. Potential participants were recruited by telephone contact beginning two weeks later to explain the study, which involved an hour-long telephone interview and blood donation for OC levels. One-hundred fifty-one daughters (70.9\%) birthed by 84 mothers (83.1\%) agreed to participate.

\subsection{Interviews}

Interviews ascertained basic information regarding birth date, birth order, and body mass index (BMI). After defining sport-caught fish as any fish eaten from a Michigan lake or river as opposed to purchased in a store or eaten in a restaurant, we asked questions regarding whether or not sport-caught fish was consumed, and for those who answered affirmatively, the ages and number of years of consumption, the types (species) of fish eaten (from a list of prompts), methods of preparation of fish eaten in the previous 12 months, and whether or not sport-caught fish had been consumed in the past year. These questions were developed by a team of investigators at this institution, including public health officials from Michigan, and had been used in previous studies of this cohort. Other variables were collected, all of which were confirmed in the parental database (in 2001/02) or using Michigan birth records, and included birth weight, whether or not the offspring was breast-fed, and if breast-fed, for what duration (in months). For each adult daughter we also determined the number of her pregnancies and the duration that each child of hers was breast-fed.

From hereon, references in this manuscript to "fish consumption" refer to sport-caught fish consumption. 


\subsection{Determination of parental DDE and PCB levels}

Non-fasting whole blood samples for DDE and PCB levels were collected in two $10 \mathrm{~mL}$ BD Vacutainers ${ }^{\circledR}$, centrifuged, and the serum placed in glass tubes and stored at $-20^{\circ} \mathrm{C}$. Blood was collected at the three time intervals referenced above and analyzed at the MDCH Health Risk Assessment Laboratory (Lansing, MI) using a modified Webb-McCall packed column gas chromatography technique to measure serum DDE and PCB levels (Hovinga et al., 1992; Price et al., 1986). The Aroclor 1260 standard was applied to determine total PCBs, as it was available for all samples; PCB congeners were not available in the parental generation. If the measured PCB level was less than the detectable limit for Aroclor $1260(3 \mu \mathrm{g} / \mathrm{L})$, we assigned a value of $1.5 \mu \mathrm{g} / \mathrm{L}$. More than $95 \%$ of the blood samples were above the limit of detection during all three sampling intervals. DDE was not determined in the first survey (1973-1974). Therefore, for maternal DDE measurements, we focused on levels measured in two intervals: 1979-1982 and 1989-1991. No DDE levels were reported to be less than the detection limit of $1 \mu \mathrm{g} / \mathrm{L}$ during these two time frames. Since serum lipid levels were not available for the parental cohort, maternal levels of DDE and PCBs were not adjusted for lipids. For these packed column studies, the between-batch precision as coefficients of variation for the lowest control was $9 \%$ for Total PCB at $12 \mathrm{ppb}$ and $6 \%$ for DDE at $10 \mathrm{ppb}$. Similarly the mid control CVs were $6 \%$ for Total PCB at $32 \mathrm{ppb}$ and $11 \%$ for DDE at $20 \mathrm{ppb}$. High control CVs were $8 \%$ for Total PCB at $95 \mathrm{ppb}$ and $2 \%$ for DDE at $60 \mathrm{ppb}$.

\subsection{Estimation of gestational levels of DDE and PCBs}


Backward extrapolation methods were used to estimate the gestational levels of DDE and PCB exposure. For 1973-1974 we had 30 measurements, for 1979-1982 we had 237, and for 1989-1991 there were 178 measurements. Detailed methodology was published previously (Karmaus et al., 2004). Briefly, we estimated two regression models covering separate periods, from 1979 to 1991 and 1973 to 1982 . Repeated serum measurements and survey information were used for the models and the regression coefficients for the most parsimonious models were generated. The results of the models were compared with how well the two equations predicted actual serum DDE and PCB concentrations as measured in the past using intraclass correlation coefficients (ICCs) (Armstrong et al., 1992). We identified two formulas that predicted past values of PCB levels with high validity (ICC $=0.77$ for the period of 1979 to 1991 , and ICC $=0.89$ for the period of 1973 to 1982). The formulas for the backward extrapolation calculations for PCBs for the two time periods are provided in Appendix, Table A.1.

These equations were then used to extrapolate maternal OC levels at the time of each pregnancy. Since the backward estimations for DDE showed only minor differences from those for PCBs, we applied the same backward estimation formulas determined for PCBs to DDE estimates. Of note from the first formula is that the predicted values were higher for 1979/82, as indicated by the positive sign for the years that passed between 1989/91 and 1979/82. The second formula has a negative sign for the years passed between the two determinations, which indicated lower predicted PCB values before 1979/82. Therefore the estimations mirror the trends that were detected for PCB concentrations in fish, which peaked around 1970 but with a delay of approximately 10 years in humans (Karmaus et al., 2004). These models did not include half-lives as a predictor for PCBs, since wide variation regarding half-life data has been reported in the literature (Shirai and Kissel, 1996). We applied the slopes of PCB and DDE over time 
derived from these two periods to backward-estimate both the maternal serum DDE and PCB concentrations at the time of each pregnancy. The average time period between the last measurement and the birth of the offspring was 16.8 years (5-95\% values: -2.1 to 30.9 years).

\subsection{Determination of adult daughters' DDE and PCB levels.}

Whole blood for the analysis of DDE and PCBs was collected and the serum was stored as described above. DDE and individual PCB congeners were extracted and measured by the Analytical Section Chemistry Laboratory at MDCH using high-resolution gas chromatography with electron capture detection according to modifications of the procedure reported previously (Mullin et al., 1984; Najam et al., 1999). Seventy-three PCB congener peaks were identified using a dual column approach. The limit of detection (LOD) for each congener was $0.03 \mu \mathrm{g} / \mathrm{L}$ for the highest sensitivity of detection and $0.125 \mu \mathrm{g} / \mathrm{L}$ for the lowest. The total PCB concentration was calculated as the sum of the PCB congeners at or above the respective LOD. The LOD was $0.125 \mu \mathrm{g} / \mathrm{L}$ for DDE. The lipid content in serum samples was determined with gravimetric methods. For these dual capillary column studies, the between-batch precision as coefficients of variation for the low control were $22 \%$ for DDE at $0.84 \mathrm{ppb}$ and $25 \%$ for Total PCB at $40 \mathrm{ppb}$ (22+1\% for PCB074 and PCB138163 at 0.8 ppb, PCB118, PCB153, and PCB187 at 0.4 ppb, and PCB180 at $0.22 \mathrm{ppb})$. Similarly the high control CVs were $16 \%$ for DDE at $1.8 \mathrm{ppb}$ and $18 \%$

for Total PCB at $90 \mathrm{ppb}(16+3 \%$ for PCB074 and PCB138163 at $1.9 \mathrm{ppb}, \mathrm{PCB} 118, \mathrm{PCB} 153$, and PCB187 at $0.9 \mathrm{ppb}$, and PCB180 at $0.47 \mathrm{ppb})$.

\subsection{Statistical Analyses}

Linear mixed effect models that take into account offspring associations resulting from sharing the same mother (nested approach) were used to evaluate the effects of estimated gestational maternal serum levels of DDE and PCBs on adult daughter OC concentrations. We 
evaluated the effects of fish consumption variables for which data are available. We found that "number of years of sport fish consumption" and "sport fish consumption in the past year" were the two most important fish intake measures for predicting the daughters' OC levels, as opposed to other measures such as the methods of preparation, body of water source, species of fish consumed, months of consumption, or even estimated number of fish consumption meals.

Because DDE and PCB levels were found to be correlated, we tested each separately in order to avoid the potential issue of multicollinearity. As a basic starting model, we considered a simple linear mixed model for which the effect of past maternal exposure on the offspring's adult exposure was assumed to be fixed and the association in the data was captured by the underlying family random effects (random intercept) term. Beforehand, a Box-Cox transformation was used for data stabilization purposes (Box and Cox, 1964). In our computations, a Box-Cox transformation was used only for the dependent variables (the DDE and PCB levels in adult daughters). All mixed models were adjusted for potential confounders in daughters, including age (in years), birth order, birth weight $(\mathrm{kg})$, length of time they were breast-fed (in months), number of pregnancies, the length of time of breastfeeding of their own children (in months), last year fish-eating status (yes/no), BMI, and lipid weight. BMI was determined using the following formula: weight $(\mathrm{kg}) /[\text { height }(\mathrm{m})]^{2}$.

OC concentrations were modeled using lipid weight as a covariate to avoid the potential bias induced by directly dividing OC exposure measurements by the lipid weight (Schisterman et al., 2005). Potential interactions among explanatory variables were also examined. All standard errors were computed using a sandwich estimator to adjust for any misspecification of the association structure implied by the working model (Fitzmaurice et al., 2004). The familial association of the offspring's adult DDE or PCB levels was estimated by the ICC. This 
coefficient can be used not only to describe the strength of association among offspring's data, but also to represent the proportion of variability explained by the mother relative to the total variability of the data of the offspring. We also used partial $\mathrm{R}^{2}$ (Edwards et al., 2008) to express the strengths of the associations between the offspring's OCs and the fixed effects such as age, BMI, and years of fish in the linear mixed effects model. The interpretation of this partial $\mathrm{R}^{2}$ is similar to that of its counterpart in the classical linear regression models for independent data.

To differentiate between maternal chemical transfer and the effects of a shared environment (e.g. similar genetics, food and other environmental exposures shared by a family unit), we analyzed data on a subset of participants whose fathers had OC concentrations measured at the same time as the birthmothers. Acknowledging that maternal and paternal levels are correlated and to avoid issues of multicollinearity in the mixed model, we transformed the OC concentration values and created two new variables. The first represented the sum of the standardized maternal and paternal OC concentrations ( $\mathrm{z}$ value), and the second, the difference between the maternal and paternal standardized values. We reasoned that the sum of the two standardized values is a variable that represents an estimate of maternal chemical transfer as well as the effect of the shared environment of the family unit, whereas the standardized paternal levels subtracted from the maternal levels is a variable that represents an estimate of the daughter's in-utero exposure and therefore the chemical transfer between mother and daughter. These two new variables were then included in the linear mixed models along with the other covariates. All statistical analyses were conducted using the SAS (Statistical Analysis System) software (version 9.2, Cary, NC). To evaluate conservatively, a two-sided p-value was used to test for statistical significance even though our hypothesis was unidirectional. 


\section{Results}

We used complete data from 132 of 151 female offspring from the Michigan Fisheaters' Cohort in this investigation, birthed by 84 different mothers. This represented $62 \%$ of the eligible population of daughters $(n=213)$. The median age of the participants was 40.4 years (range 18.4 to 65.4$)$. The median birth order was $2^{\text {nd }}$ and the median birth weight was $3.39 \mathrm{~kg}$. The median length of breastfeeding and fish consumption was 0 months and 25 years, respectively. Among the adult daughters who were breast-fed (64 out of 129), the median duration was 3 months and the $5 \%$ percentile and $95 \%$ percentile were 0.46 and 12 months, respectively. Of 132 participants, 120 (90.91\%) had eaten fish in the last year. Other characteristics of the study participants, including lipid-adjusted/unadjusted DDE and PCB levels, and maternal and estimated prenatal DDE and PCB levels are described in Table 1.

Backward extrapolation was used to estimate the gestational chemical exposure in the offspring, as described above. Due to their persistence and as a consequence of the estimation, estimated prenatal DDE levels and maternal DDE levels measured during time frames of 19791982 and 1989-1991 were highly correlated $\left(\mathrm{r}_{\text {Spearman }}=0.92, \mathrm{p}<0.001\right.$ and $\left.\mathrm{r}_{\text {Spearman }}=0.68, \mathrm{p}<0.001\right)$. Similarly, estimated prenatal PCB levels and maternal PCB levels during the two time frames were also correlated $\left(\mathrm{r}_{\text {Spearman }}=0.83, \mathrm{p}<0.001\right.$ and $\left.\mathrm{r}_{\text {Spearman }}=0.59, \mathrm{p}<0.001\right)$. Since the prenatal and maternal levels were highly correlated, we tested the models with prenatal and maternal levels separately to avoid the issue of multicollinearity.

Results for the association between adult daughter's DDE levels and prenatal or maternal DDE levels, adjusted for age, birth order, birth weight, length of breastfeeding, years of fish consumption, last year fish-eating status, number of daughter pregnancies, the length of time of breastfeeding of her own children, and lipid weight are shown in Table 2. Controlling for the 
above confounders and for intra-familial associations, DDE concentrations in adult daughters were significantly positively associated with estimated prenatal DDE levels (slope coefficient estimate $0.034, \mathrm{p}=0.01$ ). This was also true when we alternatively used the lipid weight as a covariate in the model and tested for associations between adult daughters and maternal DDE levels measured during the period of 1979-1982 (slope coefficient estimate 0.019, p<0.01) and the period of 1989-1991 (slope coefficient estimate 0.026, $\mathrm{p}=0.01$ ). The estimated models (Table 2) showed that the proportion of variability explained by the estimated prenatal and maternal DDE levels relative to the total variability of offspring's data was approximately $23 \%$.

A similar analysis for the investigation of the associations of total PCBs between mothers and daughters is shown in Table 3. In comparison to DDE, the PCB concentrations in adult daughters were more strongly positively associated with prenatal PCB levels (slope coefficient estimate 0.078, $\mathrm{p}<0.001$ ) as well as with maternal PCB levels measured during both time periods (1979-1982: slope coefficient estimate 0.043, p<0.001; 1989-1991: slope coefficient estimate $0.048, \mathrm{p}<0.001)$

Because a Box-Cox transformation was applied on the adult daughters' OC concentrations, the interpretation of regression coefficients (e.g., the coefficient of prenatal PCB level) is not trivial. To obtain easily interpretable coefficients, a Taylor expansion was used to approximate the average adult daughters' OC concentrations on the original scale $(\mu \mathrm{g} / \mathrm{L})$ by the inverse Box-Cox function of average transformed adult daughters' OC concentrations. As an example, for each unit increase of the prenatal PCB level $(\mu \mathrm{g} / \mathrm{L})$, the transformed PCB concentration in adult daughters increases by 0.078 on the Box-Cox scale (Table 3 ) which is approximately an increase of 1.081 on the original scale $(\mu \mathrm{g} / \mathrm{L})$. 
PCB concentrations in adult daughters were significantly positively associated with daughters' years of fish consumption when prenatal PCB levels were used as a predictor variable (slope coefficient estimate $0.022, \mathrm{p}<0.01$ ). This was also true for PCB concentrations when maternal PCB levels measured in the periods of 1979-1982 and 1989-1991 were used as predictors. The estimated models (Table 3) showed that the proportion of variability explained by the maternal PCB levels relative to the total variability of offspring's data is approximately $40-60 \%$.

In models with maternal PCB levels measured between 1979 and 1982 used as predictors, the daughters' fish consumption in the past year was significantly associated with the adult daughter's PCB concentrations (slope coefficient estimate $0.387, \mathrm{p}=0.03$, Table 3 ). The same relationship was found when maternal PCB levels measured between 1989 and 1991 were used as predictors (slope coefficient estimate $0.531, \mathrm{p}=0.03$ ). In the case of DDE, none of the analyses demonstrated an association between adult daughter's DDE concentrations and consumption of fish in the past year (Table 2). In addition, the main effect of years of fish consumption failed to be significantly associated with an adult daughter's DDE concentrations, using either prenatal DDE levels or maternal DDE levels as a predictor.

For both DDE and PCB concentrations in adult daughters, the daughter's age, possibly representing both an age and a birth cohort effect, was one of the remaining covariates that was significantly associated with increased OC concentrations. Both DDE and PCB concentrations in adult daughters were significantly positively linked with age (Tables 2 and 3).

BMI was not significantly related to DDE concentrations in adult offspring whenever prenatal DDE levels or maternal DDE levels were used as predictors, but were significantly associated with an adult daughter's PCB concentrations whenever maternal PCB levels measured 
in 1979-1982 or 1989-1991 were used as predictors (slope coefficient estimates were -0.162 $(\mathrm{p}=0.02)$ and $-0.187(\mathrm{p}=0.01)$, respectively).

Birth weight was significantly associated with an adult daughter's DDE concentrations, with the exception that insignificant results were found for the latter when maternal DDE levels measured between 1979 and 1982 were used as the predictor variable. Regarding adult daughters being breast-fed, there were no associations with an adult daughter's DDE or PCB concentrations (Tables 2 and 3). In contrast, the total length of time that the adult daughter breast-fed her children statistically significantly reduced her serum concentration of DDE and PCBs (Tables 2 and 3). However, there were no associations between a daughter's DDE or PCB concentrations and her total number of pregnancies.

We further explored the associations between the 73 individual PCB congeners in the daughter and maternal PCB levels measured in the period of 1979-1982. The results are shown in Appendix, Table A.2 and demonstrated that the maternal PCB levels measured during this time frame were positively associated with PCB IUPAC \#138+163, \#153, \#156, \#170, \#180, \#187, $\# 196+203$ and \#201 (only statistically significant models are presented).

Differentiating between maternal exposure (chemical transfer) and shared environment exposures (paternal chemical concentrations) using the methods described in the statistical analysis section, we found that maternal DDE concentrations measured in the period of 19791982 were more important than shared environment contributions $(n=53)$ for predicting the offspring's DDE levels in 2006-2007, as indicated by the positive estimate which is statistically significant. However, chemical transfer and shared environment measured between 1989 and 1991 became equally important for predicting offspring DDE levels in 2006-2007 ( $\mathrm{n}=37)$, as indicated by an insignificant estimate shown in Table A.3 in the Appendix. For PCBs, the 
maternal PCB concentrations measured in both of the periods of 1979-1982 ( $n=53)$ and 1989$1991(n=37)$ were equally as important as the paternal PCB concentrations measured in the same periods for predicting the offspring's PCB levels in 2006-2007 (results are given in Appendix, Table A.3).

\section{Discussion}

To our knowledge, this is the first study to report associations between maternal and adult daughter OC levels when offspring were exposed in utero. After adjusting for lipid weights and other confounders, including the shared environment, we found that 40 years later, on average, gestational concentrations of DDE and PCBs were significantly associated with higher DDE and PCB concentrations of the adult daughters.

Consideration of the toxicokinetics of the OCs studied bears comment. Wolfe et al (Wolff et al., 2007) have eloquently discussed the misclassification challenges with the estimation of exposure to these substances based on pharmacokinetic variability that is dependent on a multitude of factors, including BMI, birth cohort of exposure, genetic variability of metabolism, and rate of elimination, which vary depending on the subject's age and the magnitude of exposure, among other factors. Although the half-life in humans of both DDE and total PCBs is estimated to be 10-15 years (Ritter et al., 2011; Wolff et al., 2007), the actual halflives vary widely, depending on all of the factors listed above (El-Shahawi et al., 2010; Grandjean et al., 2008; Ritter et al., 2011; Verner et al., 2012; Wolff et al., 2007). For total PCBs, there are additional concerns because of differing elimination of the 209 congeners. While most of the body burden in the daughters that came directly from their mothers would 
have been excreted by age 40, enough remained to allow detection of the relationship with early exposure.

An important consideration in a study such as this is the differentiation between maternal in-utero chemical transfer as opposed to the effects of a shared environment (e.g. similar food and other environmental exposures shared by a family unit), and other sources of exposure during adulthood. Elimination patterns would also ideally be estimated. Because half-life data has been reported to be uncertain and is subject to varying physiological mechanisms among individuals, elimination estimates lead to varying and uncertain results. We therefore confined our estimates to those related to the shared environment. To do so, we studied the subset of participants whose fathers also had OC concentrations measured simultaneously with maternal levels. Maternal DDE concentrations measured between 1972-1982 were more predictive of adult daughter DDE levels than was the shared environment as measured by paternal OC levels, but maternal DDE levels measured later, as well as maternal PCB levels in both time periods, were equally as important predictors of offspring OC levels as was the shared environment estimation. Although these findings are intriguing, the sample sizes of the paternal OC level subgroups limit firm conclusions.

Whenever a cohort study includes a long observation period (approximately 40 years) and two generations, selection biases may distort the findings. We therefore compared the DDE and PCB levels among mothers whose offspring participated in the study and compared them with those who did not. Serum concentrations levels were higher in the 1989-91 measurements in mothers of non-participating daughters compared with mothers of participating daughters (Table 1), although these results were not statistically significant. DDE levels measured in 197982 and 1989-91 and corresponding PCB concentrations in mothers of non-participating women 
were $7.8 \mu \mathrm{g} / \mathrm{L}, 8.7 \mu \mathrm{g} / \mathrm{L}, 10.3 \mu \mathrm{g} / \mathrm{L}$, and $9.6 \mu \mathrm{g} / \mathrm{L}$, respectively. Therefore, we believe that a selection bias is unlikely to explain the results of this study.

Because the gestational levels were based on estimates, we also tested for associations between maternal levels of DDE and total PCBs measured during 1979-1982 and 1989-1991 and adult daughter concentrations of these OCs. For maternal DDE and PCBs, all associations in the two time frames were significant. Hence, overall the originally measured maternal DDE and PCB concentrations showed similar effects on the adult daughter's OC levels, indicating that the backward estimations of intra-uterine exposure did not produce artificial associations.

Interestingly, the length of time the daughter was breast-fed, as opposed to the length of time the daughters breast-fed their own children, showed no association with adult daughter's DDE or PCB concentrations. This result may be explained in part by the observation that the length of breastfeeding in those participants who were breast-fed was low (median 3 months, 5\% and $95 \%$ percentiles were 0.46 and 12 months, respectively). In addition, only $49.6 \%(n=64)$ of the daughters in our study were breast-fed.

An interesting question is whether serum concentrations of DDE and PCB found in our sample of adult daughters are higher than in the general population in the United States. Higher levels of OCs in mothers in this cohort who consumed sport-caught fish has been demonstrated in a past report (He et al., 2001). However, several mothers in our cohort consumed little or no sport-caught fish (wives of anglers). This is reflected in the wide variation of maternal DDE and PCB values (Table 1). Hence, we did not anticipate that the values in the daughters' generation would differ much from the NHANES data from the same time period. Interestingly, the lipidadjusted median DDE and PCB values in adult daughters in our cohort collected in 2007 were higher than the respective values in National Health and Nutrition Examination Survey 
(NHANES) 2006-2007 white women aged 20 - $59(\mathrm{n}=240)$. The median concentrations of DDE were $160.8 \mathrm{ng} / \mathrm{g}$ in our cohort, compared to $134.8 \mathrm{ng} / \mathrm{g}$ as reported in NHANES. We did not have access to data addressing the sum of PCBs for NHANES 2006-2007, so we focused on PCB congeners from that database and data on the same cohort of adult daughters published previously (Warner et al., 2012). All congeners of lipid-adjusted PCB concentrations were higher in adult daughters of Michigan Fisheaters than levels reported in NHANES 2006-2007. For instance for two prevalent congeners, PCB 153 and PCB180, the respective median levels were $39.9 \mathrm{ng} / \mathrm{g}$ and $21.0 \mathrm{ng} / \mathrm{g}$ in adult daughters, compared to respective values of $31.9 \mathrm{ng} / \mathrm{g}$ and $17.2 \mathrm{ng} / \mathrm{g}$ in NHANES 2006-2007.

It has been reported that PCB concentrations using the packed column methods based on Aroclor 1260, as conducted in- the parental generation, may be up to twofold higher than those measured by mass spectrometry, as done for the offspring (Longnecker et al., 2003). However, this difference does not affect our finding regarding the association of the maternal or paternal PCB levels with the offspring PCB levels. If the Aroclor 1260 measurements are systematically higher, all maternal and paternal measurements are affected equally. Thus the estimated effect on the offspring level will not change, since the estimated slope is not affected.

It is worth noting the time trends in lipid-unadjusted DDE and PCB concentrations. For DDE, the medians of maternal DDE levels measured in 1979-82 and 1989-91, and daughter's level on average at 40 years of age were $10.40 \mu \mathrm{g} / \mathrm{L}, 6.10 \mu \mathrm{g} / \mathrm{L}$, and $0.85 \mu \mathrm{g} / \mathrm{L}$, respectively. Similarly for lipid-unadjusted PCB levels, the medians of maternal levels measured in 1979-82 and 1989-91, and daughter's level on average at 40 years of age were $8.30 \mu \mathrm{g} / \mathrm{L}, 7.00 \mu \mathrm{g} / \mathrm{L}$, and $0.67 \mu \mathrm{g} / \mathrm{L}$, respectively. This is the case even after taking into account the difference between results from packed column and mass spectrometry. These trends clearly demonstrate the 
protective effect of the governmental ban on the use of these substances in the 1970s. However, a continuing legacy of exposure disadvantage in offspring of fisheaters may have been established in the Great Lakes Region due to consumption of contaminated fish in the last century.

Populations that consume fish, particularly sport-caught fish, have long been known to have higher levels of OCs compared to populations without these eating habits. The fact that the number of years of sport fish consumption was the most important fish intake measure in predicting the daughters' OC levels in our study is likely related to the simplicity of reporting a lifetime habit, as opposed to attempting to estimate numbers of fish meals, for example, which could easily be more susceptible to inaccurate estimates. The daughters in our population had a median of 25 years of fish consumption $\left(5^{\text {th }}-95^{\text {th }}\right.$ percentile range $0-35$ years $)$. Years of fish consumption was associated with offspring PCB but not DDE levels. For DDE, this was true for any measure of fish consumption duration or a history of fish consumption in the past year. Comparing the years of consumption of sport-caught fish and maternal exposure, we calculated their equivalents. The equivalent of the impact of the median prenatal DDE level of $3.67 \mu \mathrm{g} / \mathrm{L}$ on an adult daughter's DDE levels was 20.8 years of fish consumption $(3.67 \mu \mathrm{g} / \mathrm{L} \times 0.034=20.8$ years $\times 0.006$; Table 1 and 3). Similarly, the equivalent of the impact of a median prenatal PCB level of $2.56 \mu \mathrm{g} / \mathrm{L}$ on adult daughter's PCB levels was 9.08 years of fish consumption $(2.56 \mu \mathrm{g} / \mathrm{L}$ $\times 0.078=9.08$ years $\times 0.022$; Tables 1 and 3 ).

Some PCB congeners with shorter half-lives may no longer be detectable in the daughters' serum forty years later. The association between specific adult daughter PCB congener levels (PCB IUPAC \#138+163, \#153, \#156, \#170, \#180, \#187, \#196+203 and \#201) and maternal summed PCB levels measured between 1979-1982 is interesting in that each of these congeners have long half-lives (Appendix, Table A.2). 
Strengths of this study include the use of a two-generation cohort, precise measurements of DDE and PCB congeners in a certified laboratory using sophisticated state-of-the-art methods of analysis, and use of a robust database with very little missing data. Because of the time differences and associated advances in technology, when reporting on associations of DDE and PCB levels between generations, the comparability of the techniques for measurement is an important consideration. Although the possibility exists that there are differences in the methodological results, the differences are expected to be proportional and if so, should not have affected our study conclusions. Another limitation is the lack of PCB congeners in the parental sample, because at the time of measurement, laboratory methods measured PCBs only against an Aroclor standard. In addition, we are unable to calculate lipid-adjusted DDE or PCB levels in the maternal cohort, because lipid results were not analyzed in this group.

Although we found associations between a history of fish consumption in the past year and PCB levels in the adult daughters, this finding was based on a simple yes/no response to the question and because of the potential for misclassification suggested that this conclusion should be interpreted with caution. Our study questionnaire asked for a variety of other factors which can be used as possible measures of fish consumption, including age at which the participant ate fish from Michigan waters, seasonal variation, types of sport-caught fish eaten, method of preparation, and method of cleaning. As expected, simple correlation analyses demonstrated that these variables were correlated. When additionally tested, however, total years of fish consumption and consumption in the past year showed stronger associations with adult daughter's OC exposures compared to other factors. Although not perfect measures of fish consumption, these two factors were the major variables used to measure fish consumption in our study. 


\section{Conclusions}

The Michigan Fisheaters' Study provides a unique setting for investigating the effect of maternal DDE and PCB levels on the exposure of these organochlorines in their adult offspring. The burden of DDE and PCB levels in female offspring after, on average, 40 years is associated with the in-utero exposure to these chemicals. When the shared environment route of exposure was considered, as estimated by paternal organochlorine levels in the subset with paternal measurements, the association persisted, although it was attenuated. Interpretation of adverse health effects from intrauterine exposures to persistent pollutants may need to consider this correlation if this finding is duplicated in independent studies.

\section{Appendix A. Supporting information}

Table A.1.-Table A.3.

\section{Acknowledgements}

This research was supported by a grant from the Agency for Toxic Substances and Disease Registry R01 TS000007 and R01 TS000069. We gratefully thank all participants in the Great Lakes Fisheaters' Study. We are indebteded to Susan Davis for assistance in improving the manuscript. 


\section{References}

Adetona, O., et al., 2013. Concentrations of select persistent organic pollutants across pregnancy trimesters in maternal and in cord serum in Trujillo, Peru. Chemosphere. 91, 1426-33.

Armstrong, B., et al., Principles of Exposure Measurement in Epidemiology. Monagraphs in Epidemiology and Biostatistics. Oxford University Press, Oxford, New York, Tokyo, 1992.

Bezek, S., et al., 2008. Developmental origin of chronic diseases: toxicological implication. Interdiscip Toxicol. 1, 29-31.

Box, G. E. P., Cox, D. R., 1964. An Analysis of Transformations. Journal of the Royal Statistical Society. Series B (Methodological). 26, 211-252.

Butler Walker, J., et al., 2003. Organochlorine levels in maternal and umbilical cord blood plasma in Arctic Canada. Sci Total Environ. 302, 27-52.

De Coster, S., van Larebeke, N., 2012. Endocrine-disrupting chemicals: associated disorders and mechanisms of action. J Environ Public Health. 2012, Article ID 713696.

DeKoning, E. P., Karmaus, W., 2000. PCB exposure in utero and via breast milk. A review. J Expo Anal Environ Epidemiol. 10, 285-93.

Edwards, L. J., et al., 2008. An R2 statistic for fixed effects in the linear mixed model. Stat Med. 27, 6137-57.

El-Shahawi, M. S., et al., 2010. An overview on the accumulation, distribution, transformations, toxicity and analytical methods for the monitoring of persistent organic pollutants. Talanta. 80, 1587-97.

Fitzmaurice, G. M., et al., 2004. Applied Longitudinal Analysis. John Wiley and Sons, Hoboken, New Jersey. 
Gascon, M., et al., 2013. Effects of persistent organic pollutants on the developing respiratory and immune systems: a systematic review. Environ Int. 52, 51-65.

Grandjean, P., et al., 2008. Elimination half-lives of polychlorinated biphenyl congeners in children. Environ Sci Technol. 42, 6991-6.

He, J. P., et al., 2001. Time trends in sport-caught Great Lakes fish consumption and serum polychlorinated biphenyl levels among Michigan Anglers, 1973-1993. Environ Sci Technol. 35, 435-40.

Hovinga, M. E., et al., 1992. Historical changes in serum PCB and DDT levels in an environmentally-exposed cohort. Arch Environ Contam Toxicol. 22, 362-6.

Humphrey, H., 1983. Population studies of PCBs in Michigan residents. Butterworth, Boston.

Kanja, L. W., et al., 1992. A comparison of organochlorine pesticide residues in maternal adipose tissue, maternal blood, cord blood, and human milk from mother/infant pairs. Arch Environ Contam Toxicol. 22, 21-4.

Karmaus, W., et al., 2004. Backward estimation of exposure to organochlorines using repeated measurements. Environ Health Perspect. 112, 710-6.

Langer, P., 2010. The impacts of organochlorines and other persistent pollutants on thyroid and metabolic health. Front Neuroendocrinol. 31, 497-518.

Longnecker, M. P., et al., 2003. Comparison of polychlorinated biphenyl levels across studies of human neurodevelopment. Environ Health Perspect. 111, 65-70.

Mnif, W., et al., 2011. Effect of endocrine disruptor pesticides: a review. Int J Environ Res Public Health. 8, 2265-303.

Mullin, M., et al., 1984. High Resolution PCB Analysis: Synthesis and Chromatographic Properties of all 209 PCB Congeners. Environ Sci Technol. 18, 468-476. 
Najam, A. R., et al., 1999. Analysis of a mixture of polychlorinated biphenyls and chlorinated pesticides in human serum by column fractionation and dual-column capillary gas chromatography with electron capture detection. J AOAC Int. 82, 177-85.

Porpora, M. G., et al., 2013. Placental transfer of persistent organic pollutants: a preliminary study on mother-newborn pairs. Int J Environ Res Public Health. 10, 699-711.

Price, H. A., et al., 1986. Modified multiresidue method for chlordane, toxaphene, and polychlorinated biphenyls in fish. Bull Environ Contam Toxicol. 37, 1-9.

Ritter, R., et al., 2011. Intrinsic human elimination half-lives of polychlorinated biphenyls derived from the temporal evolution of cross-sectional biomonitoring data from the United Kingdom. Environ Health Perspect. 119, 225-31.

Sala, M., et al., 2001. Levels of hexachlorobenzene and other organochlorine compounds in cord blood: exposure across placenta. Chemosphere. 43, 895-901.

Schisterman, E. F., et al., 2005. Lipid adjustment in the analysis of environmental contaminants and human health risks. Environ Health Perspect. 113, 853-7.

Shirai, J. H., Kissel, J. C., 1996. Uncertainty in estimated half-lives of PCBS in humans: impact on exposure assessment. Sci Total Environ. 187, 199-210.

Verner, M. A., et al., 2012. Toxicokinetic modeling of persistent organic pollutant levels in blood from birth to 45 months of age in longitudinal birth cohort studies. Environ Health Perspect. 121, 131-7.

Waliszewski, S. M., et al., 2001. Organochlorine pesticide levels in maternal adipose tissue, maternal blood serum, umbilical blood serum, and milk from inhabitants of Veracruz, Mexico. Arch Environ Contam Toxicol. 40, 432-8. 
Warner, J., et al., 2012. Common classification schemes for PCB congeners and the gene expression of CYP17, CYP19, ESR1 and ESR2. Sci Total Environ. 414, 81-9.

Wolff, M. S., et al., 2007. Pharmacokinetic variability and modern epidemiology--the example of dichlorodiphenyltrichloroethane, body mass index, and birth cohort. Cancer Epidemiol Biomarkers Prev. 16, 1925-30.

$\mathrm{Wu}, \mathrm{H}$., et al., 2013. Persistent organic pollutants and type 2 diabetes: a prospective analysis in the nurses' health study and meta-analysis. Environ Health Perspect. 121, 153-61. 
$\underline{\text { Table 1. Characteristics of mothers and their female offspring in the Michigan Fisheaters' Cohort }}$

\begin{tabular}{|c|c|c|c|c|c|}
\hline & & $N$ & Median & $\begin{array}{c}5^{\text {th }} \\
\text { percentile }\end{array}$ & $\begin{array}{c}95^{\text {th }} \\
\text { percentile }\end{array}$ \\
\hline \multirow[t]{13}{*}{ Daughter } & Age (years) & 132 & 40.4 & 22.5 & 54.6 \\
\hline & Body Mass Index (BMI) & 131 & 25.31 & 19.47 & 39.58 \\
\hline & Birth order & 132 & 2 & 1 & 5 \\
\hline & Birth weight $(\mathrm{kg})$ & 130 & 3.39 & 2.38 & 4.08 \\
\hline & Breast fed (months) & 129 & 0 & 0 & 12 \\
\hline & Years of fish consumption & 132 & 25 & 0 & 35 \\
\hline & Number of pregnancies & 132 & 2 & 0 & 5 \\
\hline & Breastfeeding duration (months) & 132 & 2.16 & 0 & 36 \\
\hline & Lipid weight $(\mathrm{g} / \mathrm{L})$ & 123 & 5.50 & 3.88 & 7.75 \\
\hline & Lipid-adjusted DDE (ng/g) & 123 & 160.78 & 52.95 & 481.02 \\
\hline & Lipid-unadjusted DDE $(\mu \mathrm{g} / \mathrm{L})$ & 123 & 0.85 & 0.30 & 3.09 \\
\hline & Lipid-adjusted PCBs (ng/g) & 123 & 127.74 & 3.85 & 583.76 \\
\hline & Lipid-unadjusted PCBs $(\mu \mathrm{g} / \mathrm{L})$ & 123 & 0.67 & 0.02 & 3.55 \\
\hline \multirow[t]{2}{*}{ Prenatal } & Estimated DDE level $(\mu \mathrm{g} / \mathrm{L})$ & 124 & 3.67 & 0 & 11.91 \\
\hline & Estimated PCB level $(\mu \mathrm{g} / \mathrm{L})$ & 125 & 2.56 & 0 & 12.56 \\
\hline \multirow[t]{4}{*}{ Maternal } & DDE level 1979-1982 ( $\mu \mathrm{g} / \mathrm{L})$ & 81 & 10.40 & 3.40 & 28.20 \\
\hline & DDE level 1989-1991 ( $\mu \mathrm{g} / \mathrm{L})$ & 63 & 6.10 & 1.60 & 21.40 \\
\hline & PCB level 1979-1982 ( $\mu \mathrm{g} / \mathrm{L})$ & 81 & 8.30 & 1.50 & 25.90 \\
\hline & PCB level 1989-1991 ( $\mu \mathrm{g} / \mathrm{L})$ & 63 & 7.00 & 3.10 & 24.50 \\
\hline \multirow[t]{4}{*}{ Paternal } & DDE level 1979-1982 ( $\mu \mathrm{g} / \mathrm{L})$ & 58 & 19.30 & 4.40 & 80.50 \\
\hline & DDE level 1989-1991 ( $\mu \mathrm{g} / \mathrm{L})$ & 43 & 8.30 & 2.00 & 36.40 \\
\hline & PCB level 1979-1982 ( $\mu \mathrm{g} / \mathrm{L})$ & 58 & 15.00 & 4.80 & 56.00 \\
\hline & PCB level 1989-1991 ( $\mu \mathrm{g} / \mathrm{L})$ & 43 & 10.80 & 3.50 & 47.30 \\
\hline
\end{tabular}


Table 2. Coefficients from linear mixed models of serum DDE concentration in adult daughters

\begin{tabular}{|c|c|c|c|c|c|c|c|c|c|c|}
\hline & & \multicolumn{3}{|c|}{$\begin{array}{l}\mathrm{DDE}^{\mathrm{a}}(\mu \mathrm{g} / \mathrm{L}) \\
\text { No of observations: } 113 \\
\text { No of families: } 71 \\
\end{array}$} & \multicolumn{3}{|c|}{$\begin{array}{l}\mathrm{DDE}^{\mathrm{a}}(\mu \mathrm{g} / \mathrm{L}) \\
\text { No of observations: } 117 \\
\text { No of families: } 74 \\
\end{array}$} & \multicolumn{3}{|c|}{$\begin{array}{l}\mathrm{DDE}^{\mathrm{a}}(\mu \mathrm{g} / \mathrm{L}) \\
\text { No of observations: } 93 \\
\text { No of families: } 57 \\
\end{array}$} \\
\hline & & estimate & 95\% C.I. & $\begin{array}{c}\text { Partial } \\
R^{2}\end{array}$ & estimate & 95\% C.I. & $\begin{array}{c}\text { Partial } \\
R^{2}\end{array}$ & estimate & 95\% C.I. & $\begin{array}{c}\text { Partial } \\
R^{2}\end{array}$ \\
\hline \multirow[t]{10}{*}{ Daughter } & Age (yrs) & 0.032 & $(0.021,0.044)$ & 0.530 & 0.034 & $(0.022,0.047)$ & 0.480 & 0.030 & $(0.017,0.043)$ & 0.466 \\
\hline & $\mathrm{BMI}^{\mathrm{b}}$ & -0.040 & $(-0.141,0.060)$ & 0.021 & -0.029 & $(-0.130,0.072)$ & 0.011 & -0.036 & $(-0.154,0.081)$ & 0.016 \\
\hline & Birth order & -0.056 & $(-0.131,0.020)$ & 0.068 & -0.059 & $(-0.132,0.015)$ & 0.075 & -0.039 & $(-0.138,0.059)$ & 0.026 \\
\hline & Birth weight (kg) & 0.228 & $(0.035,0.421)$ & 0.157 & 0.193 & $(-0.015,0.400)$ & 0.101 & 0.260 & $(0.078,0.442)$ & 0.258 \\
\hline & Breast fed (months) & 0.014 & $(-0.022,0.050)$ & 0.019 & 0.015 & $(-0.022,0.052)$ & 0.021 & 0.009 & $(-0.031,0.049)$ & 0.009 \\
\hline & Years of fish consumption & 0.006 & $(-0.003,0.016)$ & 0.055 & 0.009 & $(-0.001,0.019)$ & 0.097 & 0.009 & $(-0.003,0.020)$ & 0.087 \\
\hline & $\begin{array}{l}\text { Ate fish in the last year } \\
\text { (Yes/No) }\end{array}$ & 0.112 & $(-0.108,0.332)$ & 0.033 & 0.077 & $(-0.143,0.298)$ & 0.016 & 0.093 & $(-0.171,0.357)$ & 0.021 \\
\hline & Number of pregnancies & 0.021 & $(-0.071,0.113)$ & 0.007 & 0.012 & $(-0.086,0.111)$ & 0.002 & 0.053 & $(-0.036,0.142)$ & 0.056 \\
\hline & $\begin{array}{l}\text { Breastfeeding duration } \\
\text { (months) }\end{array}$ & -0.016 & $(-0.023,-0.010)$ & 0.428 & -0.017 & $(-0.024,-0.010)$ & 0.417 & -0.017 & $(-0.024,-0.011)$ & 0.553 \\
\hline & Lipid weight $^{\mathrm{b}}$ & 0.296 & $(0.166,0.426)$ & 0.409 & 0.221 & $(0.067,0.375)$ & 0.211 & 0.280 & $(0.122,0.438)$ & 0.348 \\
\hline Prenatal & Estimated DDE level $(\mu \mathrm{g} / \mathrm{L})$ & 0.034 & $(0.011,0.057)$ & 0.226 & - & - & - & - & - & - \\
\hline \multirow[t]{3}{*}{ Maternal } & $\begin{array}{l}\text { DDE level } \\
1979-1982(\mu \mathrm{g} / \mathrm{L})\end{array}$ & - & - & - & 0.019 & $(0.006,0.031)$ & 0.222 & - & - & - \\
\hline & $\begin{array}{l}\text { DDE level } \\
1989-1991(\mu \mathrm{g} / \mathrm{L})\end{array}$ & - & - & - & - & - & - & 0.026 & $(0.007,0.045)$ & 0.243 \\
\hline & ICC (95\% C.I.) & 0.310 & $(0.010,0.64$ & $5)$ & 0.29 & $8(0.093,0.63$ & 9) & 0.209 & $(0.030,0.69$ & $3)$ \\
\hline
\end{tabular}

${ }^{a}$ Adult Daughter's DDE Levels are transformed by using Box-Cox transformation. ${ }^{b} \mathrm{BMI}$ and Lipid Weight are z-scores. 
Table 3. Coefficients from linear mixed models of serum PCB concentration in adult daughters

\begin{tabular}{|c|c|c|c|c|c|c|c|c|c|c|}
\hline & & \multicolumn{3}{|c|}{$\begin{array}{l}\text { PCBs }^{\mathrm{a}}(\mu \mathrm{g} / \mathrm{L}) \\
\text { No of observations: } 114 \\
\text { No of families: } 72\end{array}$} & \multicolumn{3}{|c|}{$\begin{array}{l}\mathrm{PCBs}^{\mathrm{a}}(\mu \mathrm{g} / \mathrm{L}) \\
\text { No of observations: } 117 \\
\text { No of families: } 74\end{array}$} & \multicolumn{3}{|c|}{$\begin{array}{l}\mathrm{PCBs}^{\mathrm{a}}(\mu \mathrm{g} / \mathrm{L}) \\
\text { No of observations: } 93 \\
\text { No of families: } 57\end{array}$} \\
\hline & & estimate & $95 \%$ C.I. & $\begin{array}{c}\text { Partial } \\
R^{2}\end{array}$ & estimate & 95\% C.I. & $\begin{array}{c}\text { Partial } \\
R^{2}\end{array}$ & estimate & 95\%C.I. & $\begin{array}{c}\text { Partial } \\
R^{2}\end{array}$ \\
\hline \multirow[t]{10}{*}{ Daughter } & Age (yrs) & 0.079 & $(0.060,0.099)$ & 0.689 & 0.070 & $(0.051,0.089)$ & 0.643 & 0.058 & $(0.039,0.077)$ & 0.607 \\
\hline & $\mathrm{BMI}^{\mathrm{b}}$ & -0.119 & $(-0.269,0.030)$ & 0.078 & -0.162 & $(-0.291,-0.033)$ & 0.169 & -0.187 & $(-0.321,-0.052)$ & 0.247 \\
\hline & Birth order & -0.005 & $(-0.097,0.106)$ & 0.003 & -0.005 & $(-0.100,0.091)$ & 0.0003 & 0.011 & $(-0.094,0.116)$ & 0.002 \\
\hline & Birth weight (kg) & 0.174 & $(-0.102,0.449)$ & 0.051 & 0.125 & $(-0.156,0.406)$ & 0.025 & 0.181 & $(-0.048,0.409)$ & 0.096 \\
\hline & Breast fed (months) & 0.040 & $(-0.013,0.093)$ & 0.071 & 0.042 & $(-0.009,0.092)$ & 0.082 & 0.022 & $(-0.038,0.083)$ & 0.023 \\
\hline & Years of fish consumption & 0.022 & $(0.009,0.035)$ & 0.276 & 0.024 & $(0.009,0.038)$ & 0.261 & 0.019 & $(0.003,0.035)$ & 0.202 \\
\hline & $\begin{array}{l}\text { Ate fish in the last year } \\
\text { (Yes/No) }\end{array}$ & 0.407 & $(0.053,0.761)$ & 0.150 & 0.387 & $(0.036,0.737)$ & 0.136 & 0.531 & $(0.063,0.998)$ & 0.179 \\
\hline & Number of pregnancies & 0.003 & $(-0.117,0.122)$ & 0.0001 & -0.010 & $(-0.131,0.112)$ & 0.001 & 0.055 & $(-0.049,0.159)$ & 0.045 \\
\hline & $\begin{array}{l}\text { Breastfeeding duration } \\
\text { (months) }\end{array}$ & -0.019 & $(-0.035,-0.003)$ & 0.151 & -0.018 & $(-0.034,-0.002)$ & 0.138 & -0.017 & $(-0.031,-0.003)$ & 0.197 \\
\hline & Lipid weight ${ }^{b}$ & 0.307 & $(0.082,0.532)$ & 0.200 & 0.233 & $(-0.006,0.471)$ & 0.109 & 0.366 & $(0.137,0.594)$ & 0.302 \\
\hline Prenatal & Estimated PCB level $(\mu \mathrm{g} / \mathrm{L})$ & 0.078 & $(0.045,0.111)$ & 0.425 & - & - & - & - & - & - \\
\hline Maternal & $\begin{array}{l}\text { PCB level } \\
1979-1982(\mu \mathrm{g} / \mathrm{L})\end{array}$ & - & - & - & 0.044 & $(0.026,0.061)$ & 0.440 & - & - & - \\
\hline \multirow[t]{2}{*}{ Maternal } & $\begin{array}{l}\text { PCB level } \\
\text { 1989-1991 }(\mu \mathrm{g} / \mathrm{L})\end{array}$ & - & - & - & - & - & - & 0.048 & $(0.032,0.063)$ & 0.614 \\
\hline & ICC (95\% C.I.) & 0.277 & $7(0.071,0.65$ & 9) & 0.27 & $75(0.066,0.67$ & $3)$ & 0.10 & $8(0.002,0.89$ & $6)$ \\
\hline
\end{tabular}

${ }^{\mathrm{a}}$ Adult Daughter's DDE Levels are transformed by using Box-Cox transformation. ${ }^{\mathrm{b}} \mathrm{BMI}$ and Lipid Weight are z-scores. 